\title{
Acute compartment syndrome following sodium tetradecyl sulphate (STD) injection as a treatment modality for varicose veins
}

\author{
A. P. Weerasuriya ${ }^{1}$, R. C. Siriwardana ${ }^{1}$, W.A. N. Kanchana ${ }^{2}$, M. D. P. Pinto ${ }^{1}$, P. D. Weeraddana ${ }^{2}$, \\ S. Nishanthan ${ }^{2}$, D. S. Jayatunge ${ }^{2}$ \\ ${ }^{1}$ University of Kelaniya, Ragama, Sri Lanka \\ ${ }^{2}$ Colombo North teaching hospital, Ragama, Sri Lanka
}

Key words: Acute compartment syndrome; STD

sclerotherapy; fasciotomy

\section{Introduction}

Varicose veins are a common condition, presenting mainly in the middle-aged population, which presents as enlarged, dilated and tortuous veins. Patients present with pain on prolonged standing, ankle oedema usually in the evening, bleeding, venous eczema, stasis dermatitis, lipodermatosclerosis, and appearance of telangiectasia in the affected limbs. Due to increased venous stasis within incompetent valves, severe varicosities may lead to various complications. Etiology for primary varicose veins sums up to obesity, reduced physical activity, history of trauma, pregnancy and family history of chronic venous insufficiency.

In the absence of deep venous thrombosis, peripheral arterial diseases, hypersensitivity and immobility, sclerotherapy is considered to be one of the treatment methods for varicose veins. Complete sclerosis of the vein walls is achieved by injecting a sclerosant by Fegan's technique. This cheap, technically easy OPD procedure is known to cause headache, thrombophlebitis, pigmentation and pain over the injected site as minor complications. Acute compartment syndrome following this procedure is a much rare complication.

Acute compartment syndrome is a medical emergency which can give rise to limb amputation and even death if not intervened earlier. When the fascia surrounding the compartment limits oedema, the pressure within the compartment exceeds the perfusion pressure, it gives rise to muscle and nerve ischemia. Urgent fasciotomy is needed to relieve the pressure within the compartment [1].

\section{Case presentation}

A 43-year-old male who had no medical comorbidities had right lower limb sapheno-popliteal and perforator incompetence for which he underwent Sapheno-popliteal disconnection under spinal anaesthesia and below knee sclerotherapy under ultra sound guidance. With a ratio of $1: 4$

\footnotetext{
Correspondence: Aruna Prasanna Weerasuriya

E-mail: prasannaaruna@hotmail.com

Received: 11-06-2019 Accepted: 26-09-2019

(iD) https://orcid.org/0000-0002-4223-5065

DOI: http://doi.org/10.4038/sljs.v37i3.8612
}

with a mixture of air, $2 \mathrm{ml}$ of $3 \%$ sodium tetradecyl sulphate sclerosant was injected to right leg, the total volume being $8 \mathrm{ml}$ followed by immediate application of compression bandage and Tubigrip stocking. He was given 40mg of subcutaneous Enoxaparin preoperatively to prevent DVT.

The patient was transferred to the ward after the procedure as he had no complications and the procedure was uneventful. Six hours after the procedure, the patient presented with excruciating pain in the right calf. Acute compartment syndrome was suspected upon the clinical evaluation.

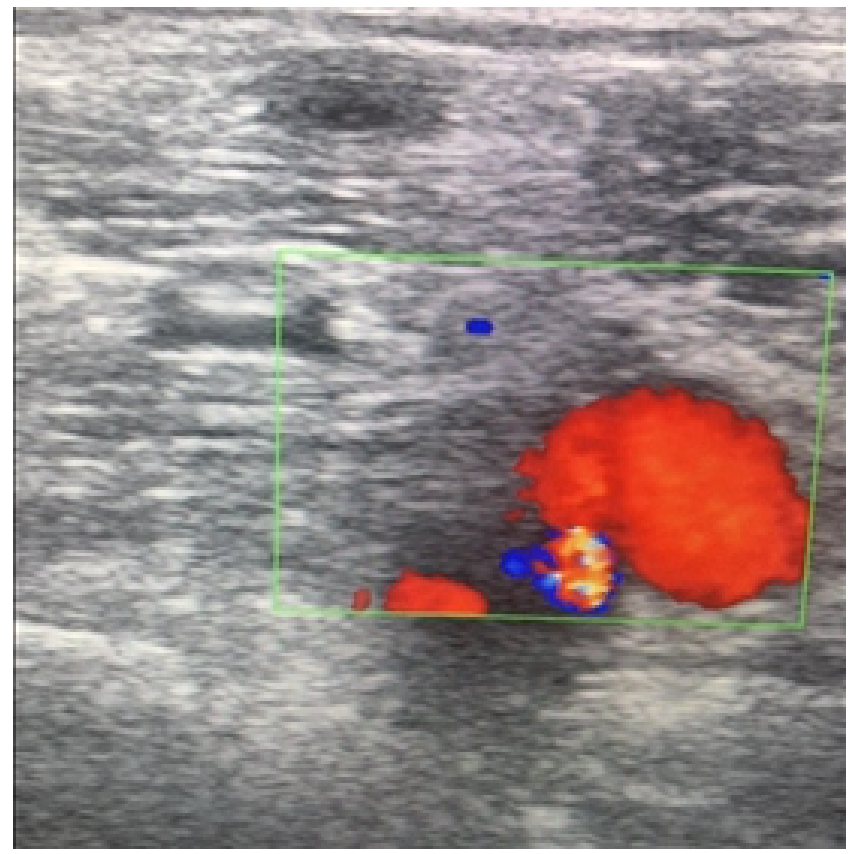

Figure 1. Duplex venous study showing cross section of popliteal vein absence of DVT

\section{Investigations}

The urgent venous duplex scan was performed upon the clinical presentation. There was no evidence of deep vein thrombosis in Femoral, Popliteal, and Tibial veins.

\section{Treatment}

In the review of rapidly progressive history of the patient, urgent two-incision fasciotomy was done under general anaesthesia, on the medial and lateral sides of the leg relieving all four compartments. 


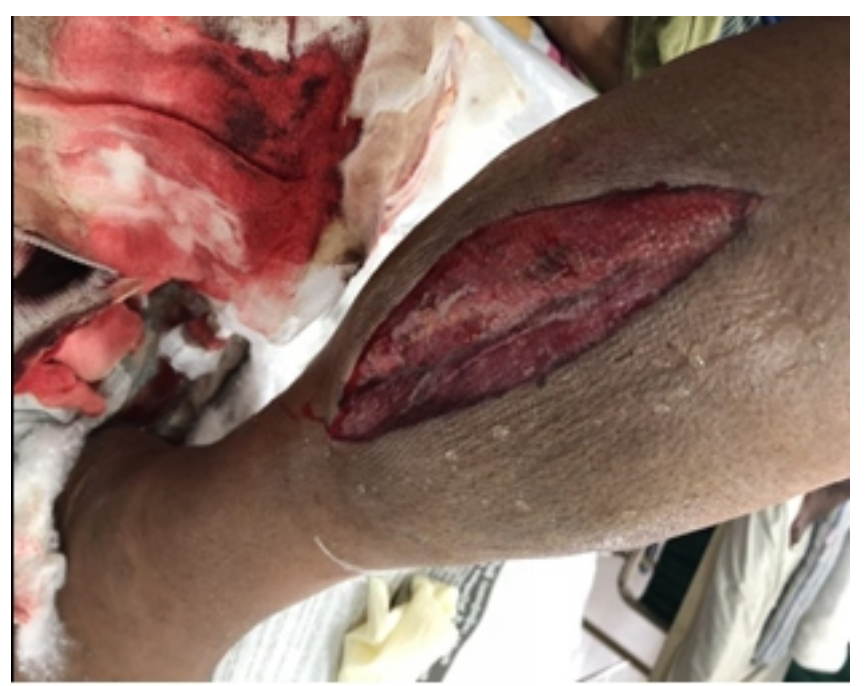

Figure 2. Lateral fasciotomy wounds day 3 post-operation

Following fasciotomy, pressure in the compartment of the leg was relieved and the patient's symptoms improved subsequently. Patient's leg kept elevated and allowed oedema to subside. On day eight patient had successful primary closure of the wound both on medial and lateral skin. He was discharged on day fifteen with no residual symptoms.

\section{Outcome/ Follow up}

With regular clinic visits and physiotherapy, the patient recovered well. Follow up scan revealed no residual symptoms.

According to a theory proposed in the 1970s, ACS leads to a reduction in arterio-venous pressure gradient and reduction in venous drainage which further results in the presentation of symptoms of the patients such as pain on passive flexion, tightness of the limbs and oedema [2]. Acute compartment syndrome is a surgical emergency where the pressure within the compartment increases out of proportion which ultimately leads to ischemia and eventually necrosis if not diagnosed early [3].

A conscious patient who can communicate well will present with symptoms such as oedema, tightness of lower limbs and pain on passive flexion. In sedated or unconscious patients, measurement of intra-compartment pressure which is above $30 \mathrm{mmHg}$ is considered critical compartment pressure [2].
Our conscious and rational patient experienced excruciating pain in the right lower limb after 6 hours, for which the surgical team intervened and took necessary actions to relieve the compartment pressure. The research suggests, if intervened after 12 hours, acute compartment syndrome damage could be irreversible. Acute compartment syndrome following injection of sclerotherapy is a phenomenon that many are unheard of. The diagnosis of ACS is clinical which can be subjective or objective, the subjective diagnosis depends solely on the clinical expertise. Progressive, persistent muscle pain which is aggravated by passive muscle stretching was the diagnostic feature of our patient. We think the dire reason behind developing ACS could be due to the microvascular impedance of the flow despite the absence of evidence of microvascular thrombi. Poor outcome in patients has been linked to delayed intervention.

The aforementioned patient's right lower limb was salvageable since the surgical team intervened immediately when the patient presented with pain. The rarity of the occurrence of ACS following sclerotherapy makes it difficult to come up with definite gold standard treatment option but, optimal outcomes were yielded when acute compartment syndrome was treated with fasciotomy as an individual entity regardless of the etiology.

All authors disclose no conflict of interest. The study was conducted in accordance with the ethical standards of the relevant institutional or national ethics committee and the Helsinki Declaration of 1975, as revised in 2000 .

\section{References}

1. Bhat SM, Rao P.General Surgery.Venous diseases, SRB's Manual Of Surgery, New Delhi, Jaypee Brothers Medical Publishers (P) Ltd, 2013; 231-235. https://doi.org/10.5005/jp/books/12831_15

2. Sidaway AN, Perler BA. Ischemia-Reperfusion. Beaulieu RJ, Grimm RJ, Hassoun HT. Rutherford's vascular surgery and endovascular therapy. Philadelphia PA. Russel Gabbedy. 2019; volume 1, pp 115-116. https://doi.org/10.1016/j.jvs.2018.08.001

3. Taylor RM, Sullivan MP, Mehta S. Acute compartment syndrome: obtaining diagnosis, providing treatment, and minimizing medicolegal risk. Curr Rev Musculoskelet Med. 2012; 5(3): pp 206-213.https://doi.org/10.1007/s12178-012-9126-y

\section{Learning Points:}

- Precautions need to be taken when using STD sclerotherapy after spinal anaesthesia because of the risk of DVT.

- Diagnose and intervene immediately when there is a high index of suspicion of acute compartment syndrome. 